\title{
Synbiotic Combinations of Lactobacillus Gasseri 505 and Cudrania Tricuspidata Leaf Extracts Ameliorate Reproductive Dysfunction in Mice Under Chronic Stress
}

\author{
Nam Su Oh ( $\sim$ klanvin@korea.ac.kr) \\ Korea University \\ Sae Hun Kim \\ Korea University \\ Jae Yeon Joung \\ Korea University \\ Whasun Lim \\ Kookmin University \\ Jiyeon Ham \\ Korea University \\ Yeon Jeong Seo \\ Korea University
}

\section{Research Article}

Keywords: chronic stress, male reproductive function, synbiotics, Lactobacillus gasseri 505 , Cudrania tricuspidata

Posted Date: January 13th, 2021

DOI: https://doi.org/10.21203/rs.3.rs-131218/v1

License: (c) (i) This work is licensed under a Creative Commons Attribution 4.0 International License. Read Full License 
6 Jae Yeon Joung, ${ }^{1}$ Whasun Lim, ${ }^{2}$ Yeon Jeong Seo, ${ }^{1}$ Jiyeon Ham, ${ }^{1}$ Nam Su Oh, ${ }^{3 *}$ and Sae Hun Kim ${ }^{1 *}$

$7{ }^{1}$ Department of Biotechnology, College of Life Sciences and Biotechnology, Korea University, Seoul, 02841,

8 Korea

$9{ }^{2}$ Department of Food and Nutrition, College of Science and Technology, Kookmin University, Seoul, 02707,

10 Korea

$11{ }^{3}$ Department of Food and Biotechnology, Korea University, Sejong, 30019, Korea

Jae Yeon Joung and Whasun Lim contributed equally to this study.

"Corresponding author: Nam Su Oh and Sae Hun Kim

E-mail: klanvin@korea.ac.kr and saehkim@korea.ac.kr

Phone: +82-2-3290-3055, Fax: +82-2-3290-3506 


\section{Abstract}

The objective of this study was to investigate the effects of Lactobacillus gasseri 505 (505) and the synbiotic combination of 505 and Cudrania tricuspidata leaf extracts (CT) on the hypothalamic-pituitary-gonadal axis in mice during chronic stress. In our previous study, a synbiotic combination (Syn) of a fermented CT-supplemented milk with 505 exhibited good anti-inflammatory and hepatoprotective activities in mice. Therefore, we hypothesized that a Syn may exert some preventive effects in the male reproductive system when suffering from unpredictable chronic mild stress (UCMS). UCMS significantly decreased the serum levels of corticosterone, however, treatment with 505 and its Syn suppressed UCMS-induced decreases. Histopathological analysis of the testes showed that these organs experienced some damage during UCMS, but this was repaired following treatment with 505 or Syn. Similarly, the transcription levels of gonadotropin-releasing hormone $(G n R H)$, GnRH receptor, and gonadotropins, moreover, testicular development (i.e., Adam5, Adam29, and Spam1) - and steroidogenesis (i.e., Lhr, Egfr, and StAR) -related genes were significantly downregulated by UCMS. These UCMS-induced changes were inhibited following the administration of 505 or Syn, which was confirmed by the results of in situ hybridization analysis. These results suggest that the administration of a Syn could attenuate the testicular dysfunctions induced by UCMS.

39 Keywords: chronic stress, male reproductive function, synbiotics, Lactobacillus gasseri 505, 
Stress is a widespread condition, with stressors becoming increasingly more prevalent in in modern society, with chronic stress causing a number of health problems. Stress-induced increases in serum glucocorticoid concentrations disrupt and suppress endocrine signaling in the male reproductive system via the hypothalamic-pituitary-gonadal (HPG) axis resulting in testicular involution. Glucocorticoid is described as the stress hormone because its levels rise sharply in response to stress. This sharp increase can result in testicular involution due to a significant drop in pituitary responsiveness to gonadotropin-releasing hormone $(\mathrm{GnRH})$ and the secretion of gonadotropins like follicle stimulating hormone (Fsh) and luteinizing hormone (Lh). The secretion of GnRH and gonadotropins are all controlled by the testicular steroids, including testosterone, estrogen inhibin and, activin all of which experience negative feedback inhibition during stress. FSH directly stimulates the Sertoli cells supporting spermatogenesis, while LH is required to stimulate the Leydig cells in the testes to secrete testosterone which acts on the Sertoli cells to aid in sperm production.

In our previous study we showed that probiotic strain Lactobacillus gasseri 505 (505), isolated from infant feces, and its synbiotic combination with Cudrania tricuspidata leaf extract (CT), one of the newly described plant-based prebiotics, improved antioxidative and anti-inflammatory activities and prevented the hepatotoxic effects associated with colorectal cancer in mice. However, the preventive effects of this synbiotic compound on male reproductive disorders resulting from chronic stress have not yet been evaluated.

Therefore, we aimed to investigate the protective effects of 505/CT synbiotics on testicular tissues during chronic stress. This study used male mice as the model organism and in order to elucidate the mechanisms of this protection we performed histopathological examinations and determined the relative transcriptional profiles of several important 
66 testicular development markers. Since there are several side effects such as sexual 67 dysfunction as well as headache, nausea, dizziness, and constipation after the clinical use of 68 antidepressants, it is necessary to develop the plant-based new therapeutics for the control of 69 reproductive disorders associated with chronic stress.

70 
Animals A total of 48 male C57BL/6J mice (8 weeks old) were purchased from Samtaco Bio Korea (Osan, Korea). The animals were maintained under a 12-hour light/dark cycle at $22 \pm$ $2{ }^{\circ} \mathrm{C}$ and RH $55 \% \pm 5 \%$ for 7 days. Mice were given access to feed (AIN-76; DooYeol Biotech, Seoul, South Korea) and tap water ad libitum during the adaptation period. The mice were randomly assigned into one of the following four treatment groups $(\mathrm{n}=12)$ : (1) control group (Con); (2) unpredictable chronic mild stress (UCMS) group (Stress); (3) probioticstreated group, treated with Lactobacillus gasseri 505 (505, $\left.10^{9} \mathrm{CFU} / \mathrm{kg} / \mathrm{day}\right)+\mathrm{UCMS}$ (Pro); and (4) synbiotic-treated group, treated with Cudrania tricuspidata leaf extract (CT)supplemented milk fermented with 505 (1,500 mg/kg/day) + UCMS (Syn). After a 7-day adaptation period, each group was fed a skim milk-based diet supplemented with each treatment for 9 weeks. UCMS was applied for 7 weeks as previously described with minor modifications . The UCMS groups were exposed to repeated mild physical and psychological stressors each day including sleep cycle changes, wet bedding, tilted cages, changes in illumination, water deprivation, restraint, and cold-water baths in randomized orders. Control groups remained undisturbed during this period except for housekeeping procedures. The mice were euthanized at week 9 using $\mathrm{CO}_{2}$ inhalation and blood was collected immediately by cardiac puncture. The brain and testes tissues were dissected and stored at $-20{ }^{\circ} \mathrm{C}$ until further physiological analysis. The overall experimental design is described in Figure 1A. All the animal experiments of this study were approved by the Institutional Animal Care and Use Committee (IACUC) of Korea University (Seoul, South Korea, approval number KUIACUC2016-182), and were performed in accordance with ARRIVE guidelines. In addition all methods were performed in accordance with the relevant guidelines and regulations. 
Preparation of the probiotics and its synbiotic compounds The probiotic strain 505, originally isolated from human infant feces, was obtained from the Korean Culture Center of Microorganisms (Seoul, South Korea, KCCM 11766P). In our previous study, this strain was determined to possess various probiotic and functional properties including acid and bile tolerance, bacterial adhesion capacity, and anti-bacterial and cholesterol reducing abilities. The genetic information for this strain can be accessed with accession number KU517710 from NCBI GenBank (https://www.ncbi.nlm.nih.gov/genbank/). This strain was sub-cultured three times in de Man, Rogosa, and Sharpe (MRS) broth (Difco, MI, USA) at $37{ }^{\circ} \mathrm{C}$ for $18 \mathrm{~h}$, prior to use. Powdered prebiotic, CT, which selectively stimulates the growth and activity of 505 and releases a number of bioactive metabolites, was added to pre-warmed milk at a final concentration of $0.2 \%(\mathrm{~g} / \mathrm{g})$. This was then pasteurized at $85{ }^{\circ} \mathrm{C}$ for $15 \mathrm{~min}$ and cooled to $41{ }^{\circ} \mathrm{C}$. This CT enriched pasteurized milk was inoculated into a $3 \%(\mathrm{v} / \mathrm{v})$ suspension of 505 (approximately $10^{7} \mathrm{CFU} / \mathrm{mL}$ ). This product was then incubated at $41{ }^{\circ} \mathrm{C}$ for $40 \mathrm{~h}$ and all samples were stored at $-20{ }^{\circ} \mathrm{C}$ before use as a freeze-dried powder.

Serum analysis Blood samples were immediately collected in BD Vacutainer ${ }^{\circledR}$ SST ${ }^{\mathrm{TM}}$ II Advance (Becton Dickinson, NJ, USA) by cardiac puncture, and serum corticosterone and serotonin levels were measured as previously described. All measurements were made in triplicate.

Histopathological Analysis For histological analysis, mice testes were fixed in $4 \%$ paraformaldehyde in phosphate-buffered saline (PBS) (pH 7.4). After $24 \mathrm{~h}$ these fixed tissues were moved to $70 \%$ ethanol for $24 \mathrm{~h}$ and then dehydrated and embedded in Paraplast-Plus (Leica Microsystems, Wetzlar, Germany). Paraffin-embedded tissues were sectioned at $5 \mu \mathrm{m}$ and stained with hematoxylin (Sigma-Aldrich, MO, USA) and eosin (Thermo Fisher, CA, 
USA). All images were captured using a Leica DM3000 microscope (Leica Microsystem Corp, Wetzlar, Germany).

Quantitative Reverse-transcription PCR The expression of mRNA was evaluated with quantitative reverse transcription PCR (qRT-PCR). Total RNA was extracted from frozen tissues as previously described. Relative gene expression was then calculated using the comparative threshold cycle method, and values were normalized against those for glyceraldehyde-3-phosphate dehydrogenase $(G A P D H)$, which acted as the internal control. The primer sequences used for qRT-PCR are listed in Table 1.

In situ hybridization analysis In situ hybridization analysis was performed as the methods of provious studies to localize Adam5, Adam29, Spam1, Lhr, Egfr, and StAR in section of the testes.

Statistical Analysis All data are shown as the mean \pm standard error standard error of the mean (SEM). Statistical significance for between-group differences was determined using one-way analysis of variance (one-way ANOVA) on SPSS version 22.0 software (IBM, 
Results

142

143

144

\section{Effects of probiotics and synbiotics treatment on serum stress markers and testicular}

histopathology To determine the physiological response to chronic stress and the effect of probiotics and synbiotics treatment, we evaluated the expression of corticosterone and serotonin and performed completed testicular histopathological analyses (Figure 1B and C). Exposure to UCMS significantly increased the serum corticosterone level compared to the Con group. However, the serum levels of corticosterone were significantly lower in the Pro and Syn groups compared to the Stress group. The serum serotonin levels dropped significantly when exposed to UCMS, but there were no significant changes in this biomarker following Pro or Syn treatment.

\section{Effects of probiotics and synbiotics treatment on the HPG axis during UCMS qRT-PCR}

was used to determine the effect of probiotics and synbiotics treatment on the transcription of those genes associated with the HPG axis (Figure 2). Exposure to UCMS significantly downregulated the expression of gonadotropin-releasing hormone $(G n R H)$ and its receptor $(G n R H r)$ at the transcript level, while treatment with the probiotics or and synbiotic compounds inhibited the UCMS-induced repression of these genes. The transcription of gonadotropins such as follicle stimulating hormone beta $(\mathrm{Fsh} \beta)$ and luteinizing hormone beta $(\operatorname{Lh} \beta)$ were also significantly decreased during chronic stress. With the transcription of these genes significantly upregulated in the Pro and Syn groups compared to the Stress group, returning their expression to normal ranges comparable to those described for the Con group.

Effects of probiotics and synbiotics treatment on the expression of testicular development-related genes during UCMS The changes in the transcription of the testes 
development-related genes in mice with UCMS are shown in Figure 3. The Stress treated group demonstrated significant differences in the expression of a disintegrin and metalloproteinase 5 (Adam5), a disintegrin and metalloproteinase 29 (Adam29), Sperm adhesion molecule 1 (Spam1), transcription factor AP-2gamma (Tcfap2c) when compared to the Con group. However, treatment with probiotics or synbiotics inhibited these UCMSinduced changes. The Syn group in particular showed significant recovery compared with the Stress and Pro groups.

Effects of probiotics and synbiotics treatment on the mRNA expression of steroidogenesis genes and growth factors during UCMS The effects of probiotics and synbiotics treatment on the expressions of steroidogenesis- and growth factor-related genes during UCMS are shown in Figure 4. In the Stress group, the Lh receptor (Lhr), epidermal growth factor receptor $(E g f r)$, and steroidogenic acute regulatory protein $(S t A R)$ were all significantly downregulated. However, inhibin alpha (Inha), steroid 5 alpha-reductase 3 (Srd5a3), and cytochrome P450 family 19 subfamily A member 1 (Cyp19al) were all significantly upregulated in stressed mice when compared to non-stressed mice. In the sample treatment groups, Pro and Syn, the abnormal expression of these genes was inhibited and their expression profiles returned to normal levels, similar to those observed in the control group. This was especially evident in Lhr, Inha, and Srd5a3 expression and these changes were more significant in the Syn group.

Localization of male reproductive transcripts in testicular tissues A detailed analysis of the male reproductive system-related genes (i.e., Adam5, Adam29, Spam1, StAR, Egfr, and Lhr) in the testes following UCMS treatment was performed using in situ hybridization (Figure 5). The expression levels of these genes were reduced in the spermatocytes (Spc), 

spermatogonia (Spg), spermatids (Spt), and spermatozoa (Spz) of mice treated with UCMS when compared to the control group. Conversely, higher expression levels for each of these genes were observed following probiotics or synbiotics treatment. The expression of Adam5, Spam1, and Lhr showed the greatest improvement in the Syn treated group. As with the 194 results of the qRT-PCR, in situ hybridization revealed that chronic stress had a negative effect 195 on the expression of the testicular reproductive genes, including testicular development 196 markers, steroidogenesis markers, and hormone/growth markers. Furthermore, these results

197 indicate that the administration of probiotic strain 505 and its synbiotics containing CT could 198 prevent the male reproductive dysfunction associated with chronic stress. 
Discussion

This study determined that synbiotics made up of Lactobacillus gasseri 505 and

Cudrania tricuspidata leaf extracts could provide a protective effect for testicular development and function during chronic stress. UCMS exerts a negative effect on the expression of reproductive-related genes in mice brains and testes, leading to deteriorations in the male reproductive system, including abnormal testicular development, steroidogenesis, transcription, and hormone/growth factor expression. In contrast, UCMS-induced reproductive dysfunctions were improved via the HPG axis following treatment with probiotic strain 505 and its synbiotics containing CT. These preventive effects may be the result of the probiotic strain 505 or the phenolic compounds and peptides derived from the fermentation of CT-supplemented milk by 505. In our previous study, 505 was shown to exhibit acid and bile tolerance, adhesion capacity, antibacterial activity, and cholesterolreducing ability. Moreover, CT contains appreciable levels of several phenolic compounds, including chlorogenic acid, caffeic acid, quercetin-3-glucoside, and 3,4-dihydroxyhydrocinnamic acid, which have all been described as exerting various antioxidant properties. In addition, various bioactive peptides (i.e., antimicrobial, antihypertensive, and antioxidative activities and ACE inhibitory activity) have been identified following the fermentation of CTsupplemented milk with 505. These bioactive compounds and probiotic strain impacted the preventive effects for chronic stress-induced testicular dysfunctions in their synbiotic combination.

The histological evaluation of the testes revealed that UCMS induced the disruption of the multilayered epithelial skeleton and diminished the number of spermatozoa in the seminiferous tubules. A significant increase in the extent of cellular damage, and thus testicular damage, was observed in the testicular sections of mice with UCMS when 
compared to the Con group. Conversely, treatment with probiotics or synbiotics showed recovery in the seminiferous tubules structure with these structures almost returning to their pre-stressed condition. These results indicate that the administration of 505 and/or its synbiotics improved stress response and reduced cellular damage in the testes. Moreover, treatment of 505 and synbiotics prevent UCMS-induced downregulation of $G n R H$ and $G n R H r$ and their downstream gonadotropins, which may help to retain reproductive function during chronic stress. GnRH is a tropic peptide hormone synthesized and released from GnRH neurons within the hypothalamus, and constitutes the initial step in the HPG axis leading to the release of Fsh and Lh from the anterior pituitary. Fsh, a glycoprotein polypeptide hormone, enhances the production of androgen binding protein in the Sertoli cells of the testes by binding to the Fsh receptors on their membranes, and is critical for the initiation of spermatogenesis. Lh stimulates Leydig cells to produce testosterone, since Lh regulates the expression of 17ß-hydroxysteroid dehydrogenase which converts androstenedione to testosterone, an androgen that exerts both endocrine and intra-testicular effects on spermatogenesis. Besides, adam family proteins expressed in male reproductive tissues undergo unique processing during sperm maturation and are located on the surface of the sperm heads. Adam5 is known to play a particularly important role in fertilization, while Adam29 is involved in transducing cellular signals related to maturation of testicular cells. Spaml encodes an enzyme located on the sperm surface and inner acrosomal membrane. This multifunctional protein is a hyaluronidase that enables sperm to penetrate the hyaluronic acidrich cumulus cell layer surrounding the oocyte, a receptor that plays a role in hyaluronic acid induced cell signaling, and a receptor that is involved in sperm-zona pellucida adhesion. Moreover, Hoei-Hansen et al. reported that Tcfap $2 c$ is involved in the undifferentiated phenotype in germ cells and could be a marker of testicular tumors. Exposure to UCMS downregulated a number of genes related to testicular development which could result in 
testicular dysfunction. However, treatment with either the probiotic strain 505 or its synbiotics with CT, especially the synbiotics, prevent the UCMS-induced changes in the expressions of these genes which may lead to protection of testicular involution. In addition, the results for the preventive effects of 505 and synbiotics on steroidogenesis were in agreement with a study conducted by Kushwaha and Gupta which showed that chronic stress had a negative effect on the male reproductive system via the HPG axis, effected by endocrine activity and Lh secretion. Lh interacts with its receptor Lhr and this activation is critical for spermatogenesis while Egfr is the prototypical member of its family of membrane associated intrinsic tyrosine kinase receptors, which are required for optimal embryonic testes converted into dihydrotestosterone (DHT), and this conversion is catalyzed by the microsomal enzyme encoded by $\operatorname{Srd5a3.~Srd5a3}$ activity is a late stage event in male sexual development, that requires the correct developmental interpretation of both genetic and hormonal signals. The overexpression of $\operatorname{Srd5a3}$ increases the levels of DHT, which causes hair loss and prostate diseases including benign prostatic hyperplasia and prostate cancer. Testosterone is also converted to estradiol by Cyp19al activating the nuclear estrogen receptor. Cyp19al is highly expressed in the undifferentiated gonads and is upregulated during initial male sexual differentiation events. These results indicate that treatment with 505 or its synbiotics could prevent the abnormal hormonal biosynthesis associated with 
275 UCMS-induced testicular dysfunctions via transcriptional regulation of key developmental 276 factors. Taken together, current study demonstrated that administration of a synbiotic 277 combination of 505 and CT attenuated the UCMS-induced alteration in testicular 278 development- and steroidogenesis-related genes, which was also confirmed with in situ 279 hybridization analysis. Consequently, this study suggested that this novel synbiotics could act 280 as a natural protective agent for male fertility during chronic stress. 
283

284

285

286

287

1. Munck, A., Guyre, P. M. \& Holbrook, N. J. Physiological functions of glucocorticoids in stress and their relation to pharmacological actions. Endocr Rev 5, 25-44, doi:10.1210/edrv-5-1-25 (1984).

2. Suter, D. E. \& Schwartz, N. B. Effects of glucocorticoids on secretion of luteinizing hormone and follicle-stimulating hormone by female rat pituitary cells in vitro. Endocrinology 117, 849-854, doi:10.1210/endo-117-3-849 (1985).

3. Hardy, M. P. et al. Stress hormone and male reproductive function. Cell Tissue Res 322, 147-153, doi:10.1007/s00441-005-0006-2 (2005).

4. Wagenmaker, E. R., Breen, K. M., Oakley, A. E., Tilbrook, A. J. \& Karsch, F. J. Psychosocial stress inhibits amplitude of gonadotropin-releasing hormone pulses independent of cortisol action on the type II glucocorticoid receptor. Endocrinology 150, 762-769, doi:10.1210/en.2008-0757 (2009).

5. Nargund, V. H. Effects of psychological stress on male fertility. Nat Rev Urol 12, 373382, doi:10.1038/nrurol.2015.112 (2015).

6. Rajkanna, J., Tariq, S. \& Oyibo, S. O. Successful fertility treatment with gonadotrophin therapy for male hypogonadotrophic hypogonadism. Endocrinol Diabetes Metab Case Rep 2016, 150124, doi:10.1530/EDM-15-0124 (2016).

7. Oh, N. S., Lee, J. Y. \& Kim, Y. The growth kinetics and metabolic and antioxidant activities of the functional synbiotic combination of Lactobacillus gasseri 505 and Cudrania tricuspidata leaf extract. Appl Microbiol Biotechnol 100, 10095-10106, doi:10.1007/s00253-016-7863-3 (2016).

8. Oh, N. S. et al. A synbiotic combination of Lactobacillus gasseri 505 and Cudrania tricuspidata leaf extract prevents hepatic toxicity induced by colorectal cancer in mice. $\mathrm{J}$ Dairy Sci 103, 2947-2955, doi:10.3168/jds.2019-17411 (2020).

9. Higgins, A., Nash, M. \& Lynch, A. M. Antidepressant-associated sexual dysfunction: impact, effects, and treatment. Drug Healthc Patient Saf 2, 141-150, doi:10.2147/DHPS.S7634 (2010).

10. Cascade, E., Kalali, A. H. \& Kennedy, S. H. Real-World data on SSRI antidepressant side effects. Psychiatry (Edgmont) 6, 16-18 (2009).

11. Oh, N. S. et al. Glycated milk protein fermented with Lactobacillus rhamnosus ameliorate the cognitive health of mice under mild stress condition. Gut Microbes, 
doi:10.1080/19490976.2020.1756690 (2020).

12. Bae, S. M. et al. Sex-specific expression of CTNNB1 in the gonadal morphogenesis of the chicken. Reprod Biol Endocrinol 11, 89, doi:10.1186/1477-7827-11-89 (2013).

13. Joung, J. Y., Lee, J. S., Oh, N. S. \& Kim, S. H. Fermented Maillard reaction products attenuate stress-induced testicular dysfunction. J Dairy Sci, doi:10.3168/jds.2020-18996 (2020).

14. Oh, N. S. et al. Improved functionality of fermented milk is mediated by the synbiotic interaction between Cudrania tricuspidata leaf extract and Lactobacillus gasseri strains. Appl Microbiol Biotechnol 100, 5919-5932, doi:10.1007/s00253-016-7414-y (2016).

15. Brown, R. An introduction to neuroendocrinology. (Cambridge University Press, 1994).

16. Boron, W. F. \& Boulpaep, E. L. Medical physiology: a cellular and molecular approach. (Saunders, W. B., 2008).

17. Louvet, J. P., Harman, S. M. \& Ross, G. T. Effects of human chorionic gonadotropin, human interstitial cell stimulating hormone and human follicle-stimulating hormone on ovarian weights in estrogen-primed hypophysectomized immature female rats. Endocrinology 96, 1179-1186, doi:10.1210/endo-96-5-1179 (1975).

18. Ramaswamy, S. \& Weinbauer, G. F. Endocrine control of spermatogenesis: Role of FSH and LH/ testosterone. Spermatogenesis 4, e996025, doi:10.1080/21565562.2014.996025 (2014).

19. Han, C. et al. Comprehensive analysis of reproductive ADAMs: relationship of ADAM4 and ADAM6 with an ADAM complex required for fertilization in mice. Biol Reprod 80, 1001-1008, doi:10.1095/biolreprod.108.073700 (2009).

20. Lim, W. et al. Gossypol induces disruption of spermatogenesis and steroidogenesis in male mice. J Agric Food Chem 67, 2075-2085, doi:10.1021/acs.jafc.8b06946 (2019).

21. Evans, E. A., Zhang, H. \& Martin-DeLeon, P. A. SPAM1 (PH-20) protein and mRNA expression in the epididymides of humans and macaques: utilizing laser microdissection/RT-PCR. Reprod Biol Endocrinol 1, 54, doi:10.1186/1477-7827-1-54 (2003).

22. Hoei-Hansen, C. E. et al. Transcription factor AP-2gamma is a developmentally regulated marker of testicular carcinoma in situ and germ cell tumors. Clin Cancer Res 10, 8521-8530, doi:10.1158/1078-0432.CCR-04-1285 (2004).

23. Kushwaha, B. \& Gupta, G. Sexually transmitted infections and male infertility: old enigma, new insights. (Springer, 2017). 
24. Levine, E., Cupp, A. S., Miyashiro, L. \& Skinner, M. K. Role of transforming growth factor-alpha and the epidermal growth factor receptor in embryonic rat testis development. Biol Reprod 62, 477-490, doi:10.1095/biolreprod62.3.477 (2000).

25. Chen, J. et al. Expression and function of the epidermal growth factor receptor in physiology and disease. Physiological Reviews 96, 1025-1069 (2016).

26. O'Connor, A. E. \& De Kretser, D. M. Inhibins in normal male physiology. Semin Reprod Med 22, 177-185, doi:10.1055/s-2004-831893 (2004).

27. Stocco, D. M. StAR protein and the regulation of steroid hormone biosynthesis. Annu Rev Physiol 63, 193-213, doi:10.1146/annurev.physiol.63.1.193 (2001).

28. Andersson, S., Bishop, R. W. \& Russell, D. W. Expression cloning and regulation of steroid 5 alpha-reductase, an enzyme essential for male sexual differentiation. J Biol Chem 264, 16249-16255 (1989).

29. Marks, L. S. 5alpha-reductase: history and clinical importance. Rev Urol 6 Suppl 9, S1121 (2004).

30. Purves-Tyson, T. D. et al. Testosterone regulation of sex steroid-related mRNAs and dopamine-related mRNAs in adolescent male rat substantia nigra. BMC Neurosci 13, 95, doi:10.1186/1471-2202-13-95 (2012).

31. Norris, D. O. \& Lopez, K. H. Hormones and reproduction of vertebrates. (Academic Press, 2010). 
Acknowledgements

369 This research was supported by the National Research Foundation (NRF) of Korea (NRF$370 \quad$ 2019R1F1A1041471.

\section{Author contributions}

372 NSO and SHK conceived and designed the research, and revised the manuscript. JYJ and HL conducted the experiments, analyzed data, and wrote the manuscript. YJS and JH conducted the experiments. All authors read and approved the final manuscript.

Additional information

377 The authors have no conflicts of interest to declare.

378

379 
381 Table 1. Details of the primers used in this study

\begin{tabular}{ccc}
\hline $\begin{array}{c}\text { Gene } \\
\text { Symbol }\end{array}$ & Forward primer $\left(5^{\prime} \rightarrow 3^{\prime}\right)$ & Reverse primer $\left(5^{\prime} \rightarrow 3^{\prime}\right)$ \\
\hline GnRH & AGCACTGGTCCTATGGGTTG & GGGGTTCTGCCATTTGATCCA \\
GnRHr & TGCTCGGCCATCAACAACA & GGCAGTAGAGAGTAGGAAAAGGA \\
Fshb & GCCATAGCTGTGAATTGACCA & AGATCCCTAGTGTAGCAGTAGC \\
Lhb & CTGAGCCCAAGTGTGGTGTG & GACCATGCTAGGACAGTAGCC \\
Adam5 & AGGAGAATCTGTGGCAATGG & CCGTGCAATCTTGACTACAGC \\
Adam29 & CCATGAATGTCCAGATGATGC & TTGCCTACAGTGCTCATTGC \\
Spam1 & GAATGGAGGCCTACCTGGTT & CTTCCTTCCTGCCTCTTCAA \\
Tcfap2c & AGAGGAGGTGCAGAATGTGG & CAGGGACTGAGCAGAAGACC \\
Lhr & CGCCCGACTATCTCTCACCTA & GACAGATTGAGGAGGTTGTCAAA \\
Egfr & GGGGATGTGATCATTTCTGG & GCCTTGCAGTCTTTCTCAGC \\
Inha & GTCTCTGCTGCTCCTTTTGC & GGAATAGAGCCTTCACCTTGG \\
StAR & TCTGCTTGGTTCTCAACTGG & TTCTGCATAGCCACCTCTCC \\
Srd5a3 & CCGCCCATCAGTATAAATGC & CTCGAACCAGTCTCCAAAGG \\
Cyp19a1 & TGTTGTGGACTTGGTCATGC & TGGGCTTAGGGAAGTACTCG \\
Gapdh & GACGGCCGCATCTTCTTGT & CAGTGCCAGCCTCGTCCCGTACAA \\
\hline
\end{tabular}


385

386

Figure 1. [A] Schematic overview of the animal experiments. [B] Effects of probiotics and synbiotics treatment on the serum concentrations of corticosterone and serotonin in mice during UCMS. Data are expressed as the mean $\pm \operatorname{SEM}(n=12)$ from three independent experiments. \# Significant difference when compared to the Con group $\left({ }^{\#} p<0.05,{ }^{\#} p<0.005,{ }^{\# \#} p<0.001\right)$. * Significant difference when compared to the Stress group $(* p<0.05, * * p<0.005, * * * p<0.001)$. [C] Representative histological sections from mice testes stained with hematoxylin and eosin (calibration bar $=200 \mu \mathrm{m})$.

Figure 2. Effects of probiotics and synbiotics treatments on the transcription of genes related to the HPG axis in mouse brains during UCMS. Data represents the relative expression of GnRH, GnRHr, Fshb, and Lhb compared to Gapdh. Data are expressed as the mean \pm SEM $(n=12)$ from three independent experiments. \# Significant difference when compared to the Con group $\left({ }^{\#} p<0.05,{ }^{\# \#} p<0.005\right.$, $\#$ \#\# $p$ 0.001). * Significant difference when compared to the Stress group ${ }^{*} p<$ $0.05, * * p<0.005, * * * p<0.001)$.

Figure 3. Effects of probiotics and synbiotics treatments on the transcription of testicular development genes in mice testes under UCMS. Data represents the relative expression of Adam5, Adam29, Spam1, and Tcfap2c compared to Gapdh. Data are expressed as the mean \pm SEM $(n=12)$ from three independent experiments. \# Significant difference compared to the Con group ${ }^{\#} p<0.05,{ }^{\# \#} p<0.005,{ }^{\# \#} p<$ 0.001). * Significant difference compared to the Stress group $(* p<0.05$, ** $p<$ $0.005, * * * p<0.001)$ 
Figure 4. Effects of probiotics and synbiotics treatments on the transcription of spermatogenesis and growth factor related genes in mice testes under UCMS. Data describes the relative expressions of Lhr, Egfr, Inha, StAR, Srd5a3, and Cyp19al compared to Gapdh. Data represent the mean \pm SEM (n=12) from three independent experiments. ${ }^{\#}$ Significant difference compared to the Con group $\left({ }^{\#} p\right.$ $\left.<0.05,{ }^{\#} p<0.005,{ }^{\# \#} p<0.001\right)$. * Significant difference compared to the Stress group $(* p<0.05, * * p<0.005, * * * p<0.001)$.

Figure 5. In situ hybridization of Adam5, Adam29, Spam1, Lhr, Egfr, and StAR in mice testes. mRNA expression was analyzed using cross-sections from mice testes and antisense or sense target gene cRNA probes. L, Leydig cells; Spc, spermatocytes; Spg, spermatogonia; Spz, spermatozoa. Scale bar represents $10 \mu \mathrm{m}$. 
[A]

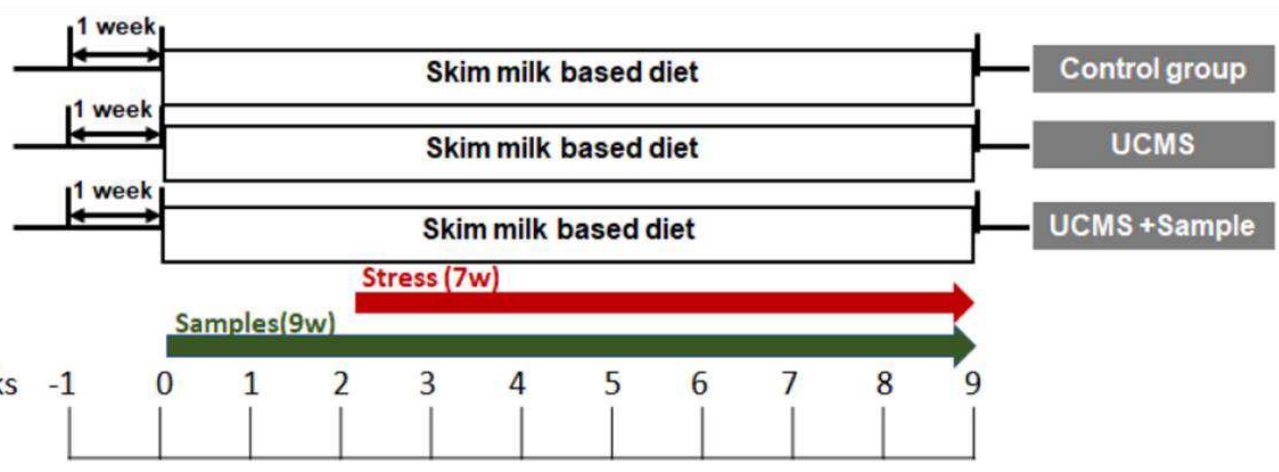

[B]
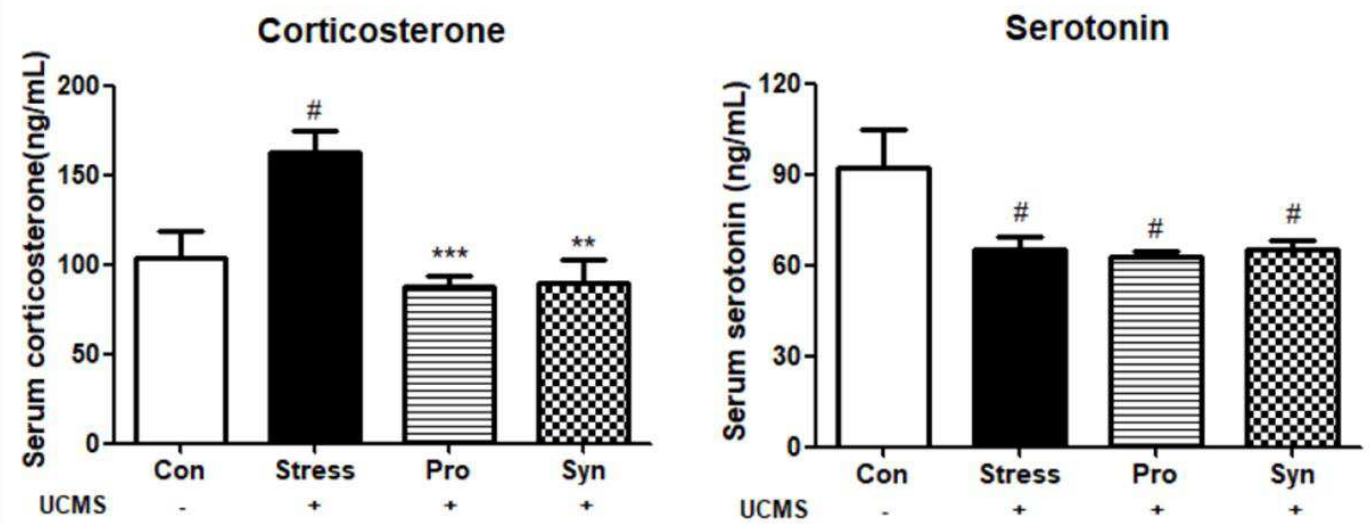

420

[C]
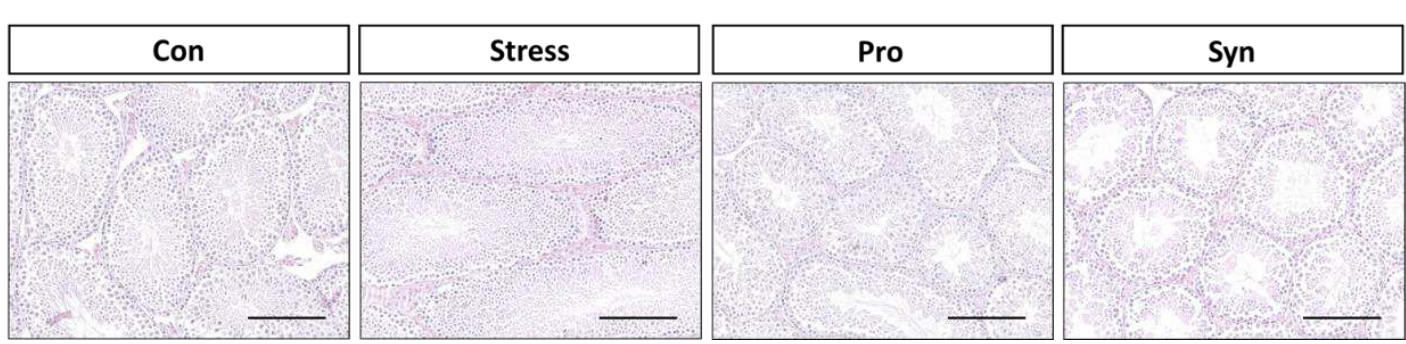

$421 \quad$ Figure 1.

422 
423
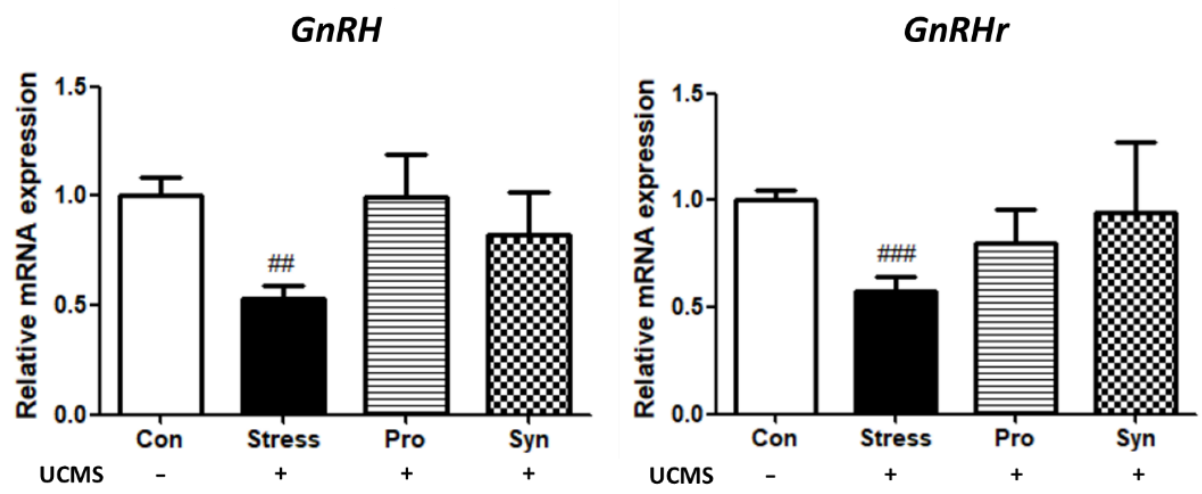

Fshb
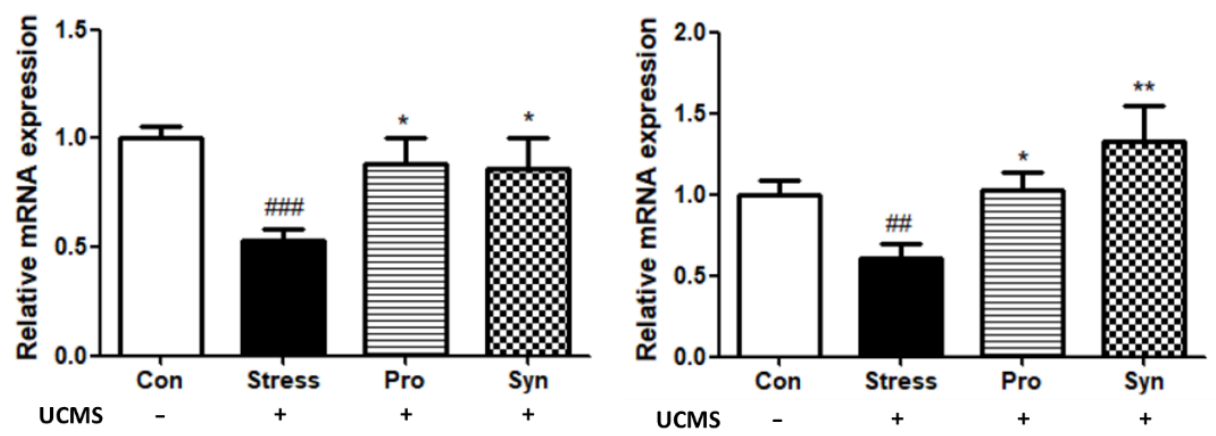

424

425 Figure 2.

426 
427

Adam5

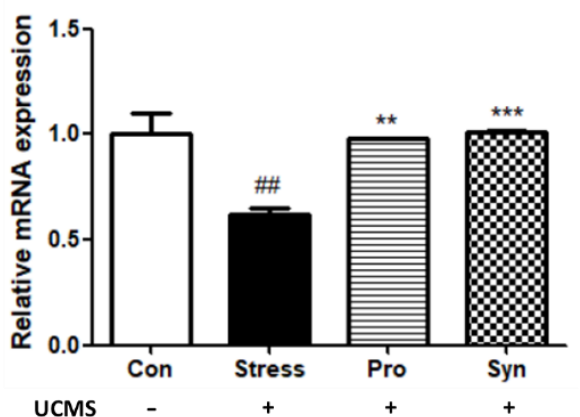
UCMS

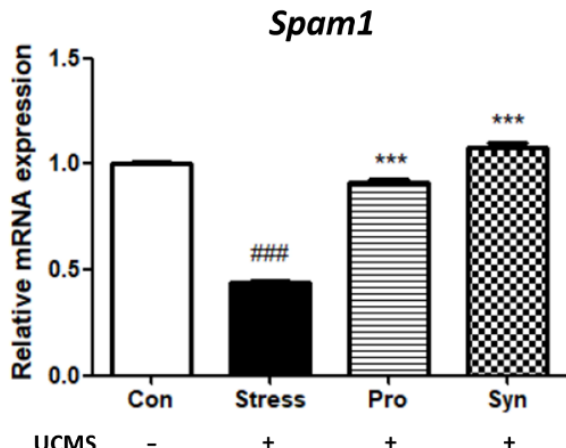

Adam29

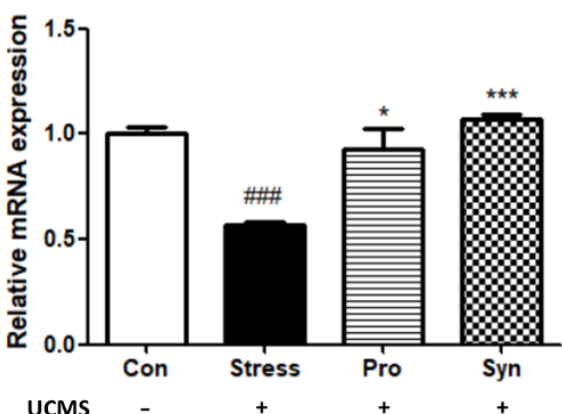

Tcfap2c

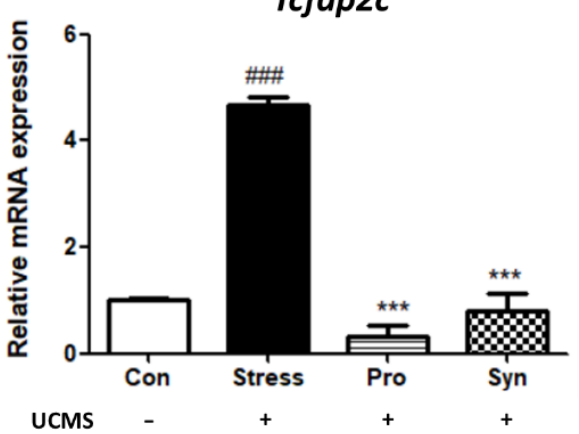



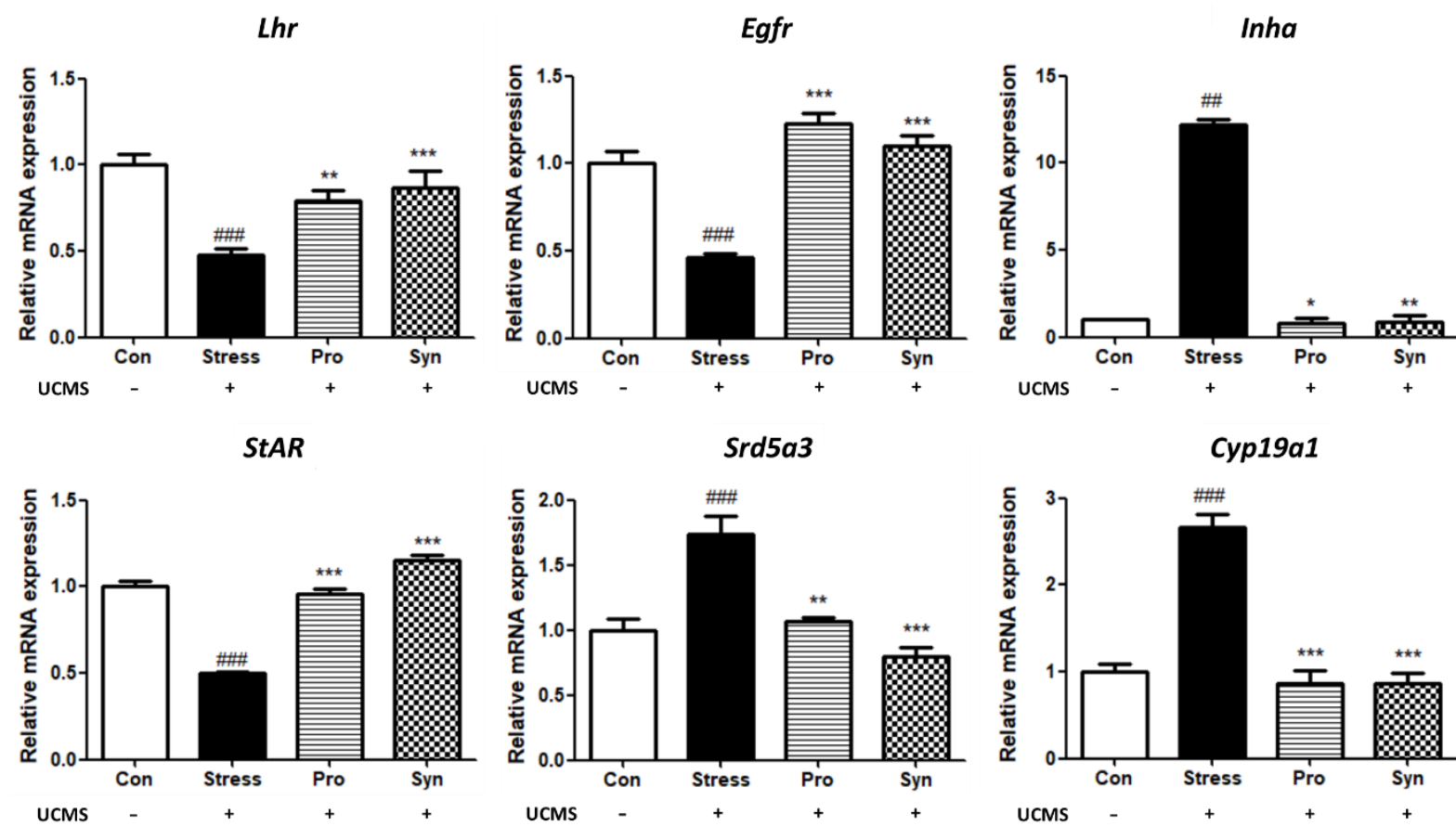

$432 \quad$ Figure 4. 


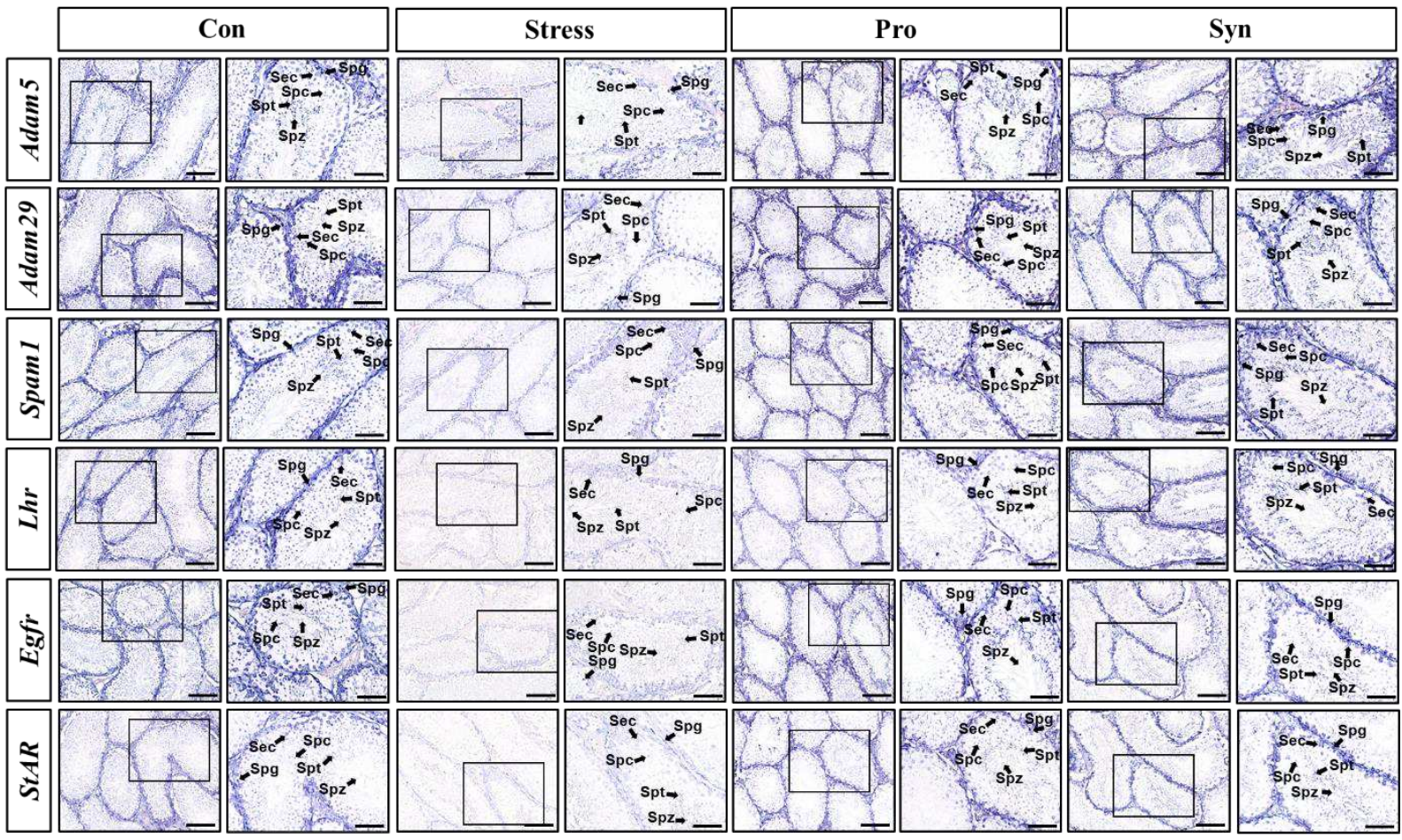

$436 \quad$ Figure 5. 


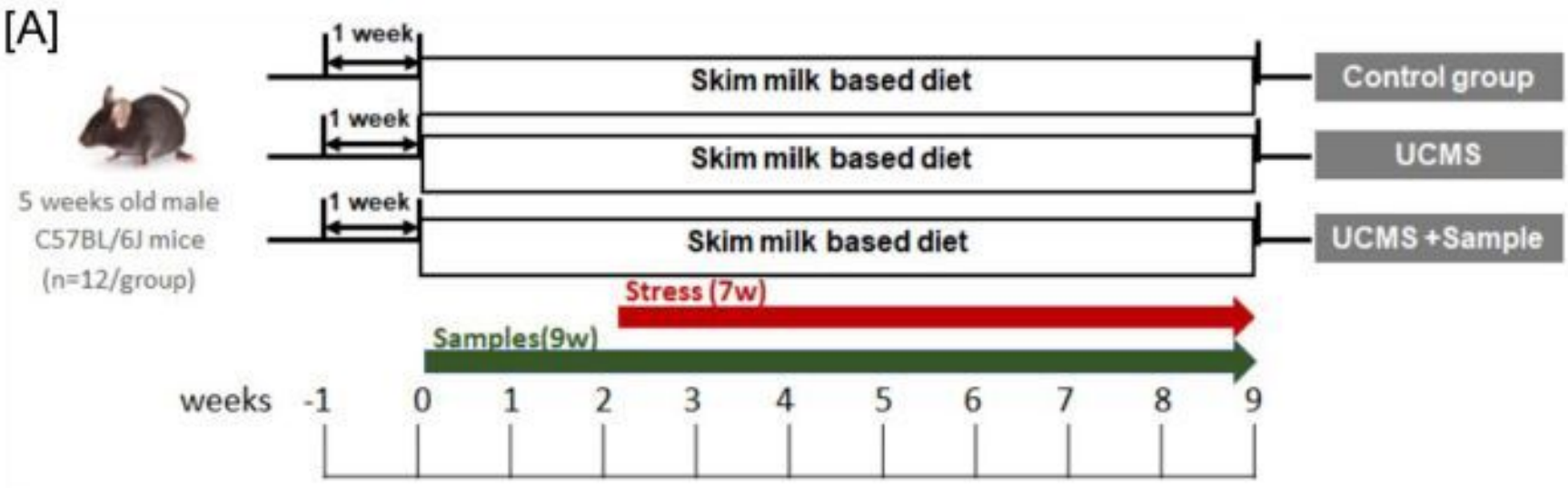

$[\mathrm{B}]$
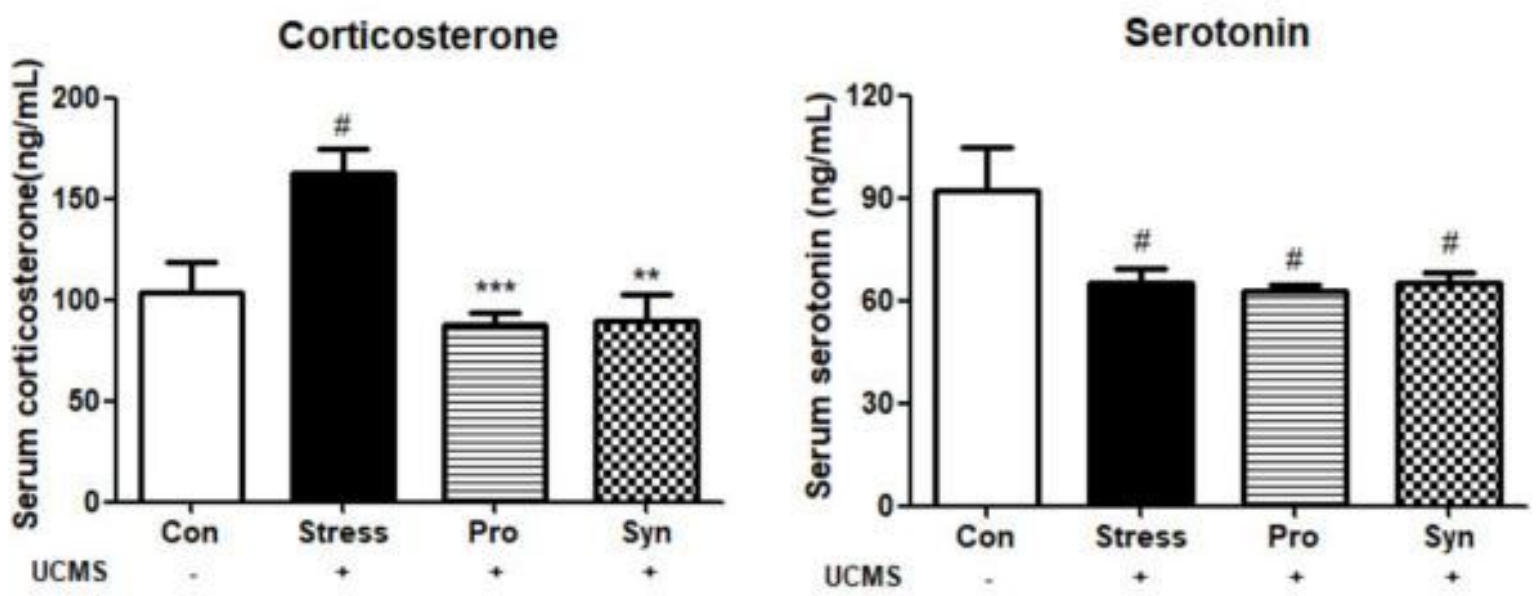

[C]
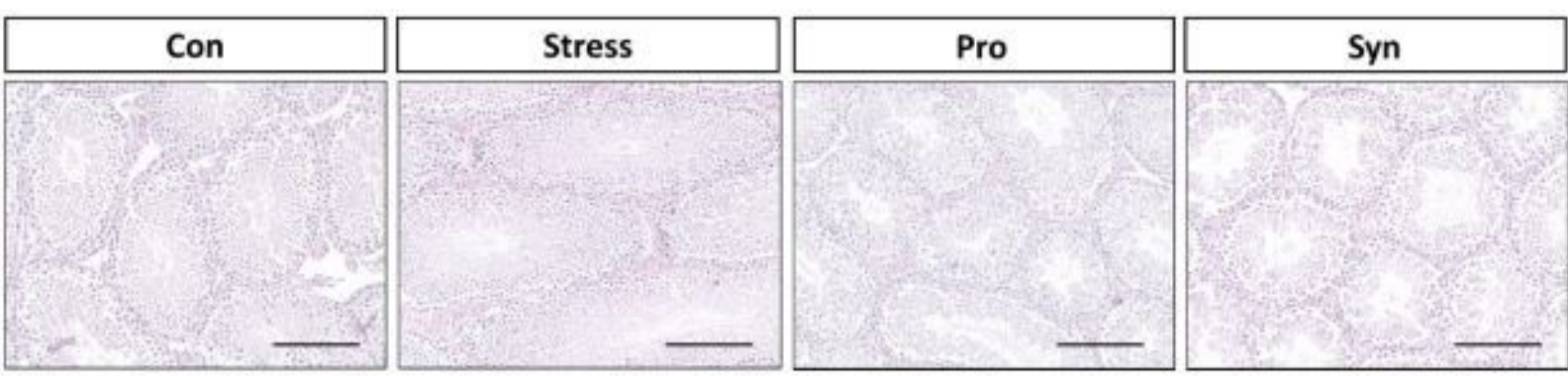

\section{Figure 1.}

\section{Figure 1}

[A] Schematic overview of the animal experiments. [B] Effects of probiotics and synbioticstreatment on the serum concentrations of corticosterone and serotonin in mice during UCMS. Data are expressed as the mean \pm SEM $(n=12)$ from three independent experiments. \#Significant difference when compared to

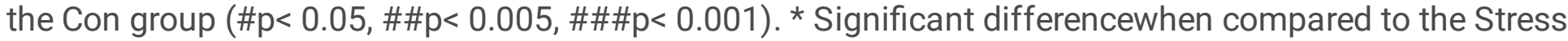


group ( $\left.{ }^{*}<<0.05,{ }^{\star *} p<0.005,{ }^{\star * \star} p<0.001\right)$. [C] Representative histological sections from mice testes stained with hematoxylin and eosin (calibration bar $=200 \mu \mathrm{m}$ ).

\section{GnRH}

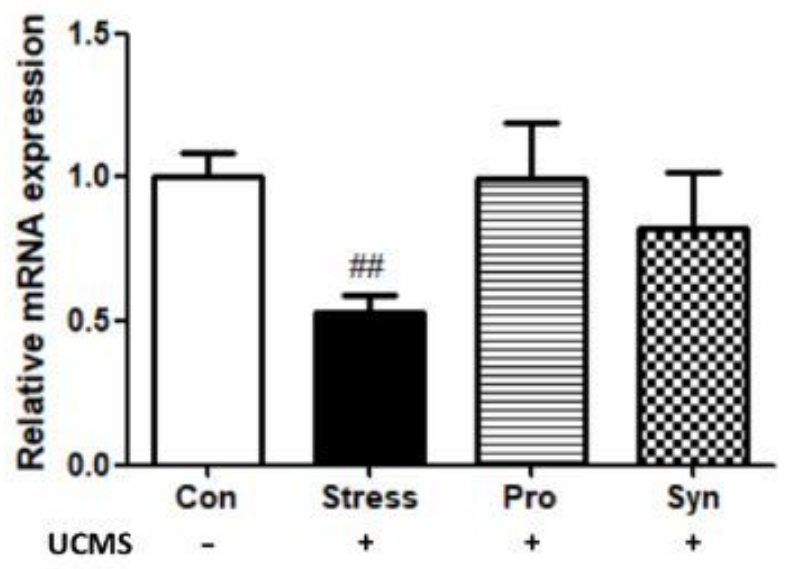

Fshb

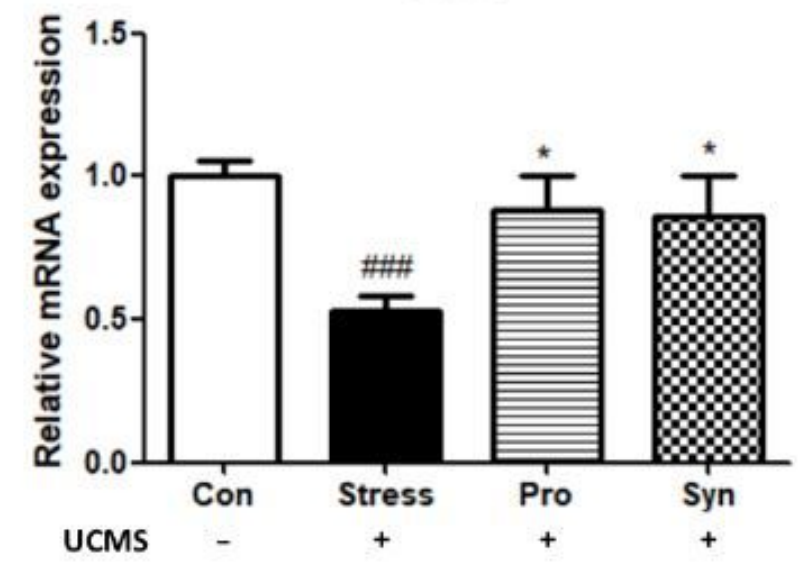

GnRHr

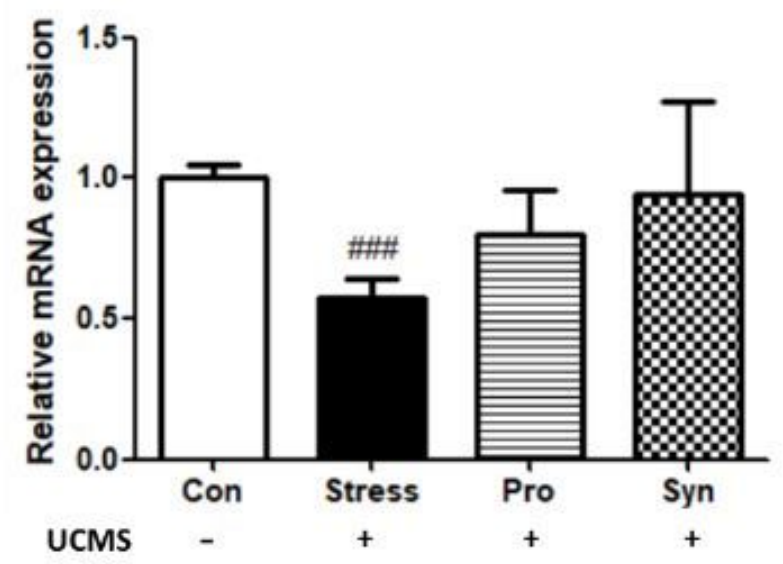

Lhb

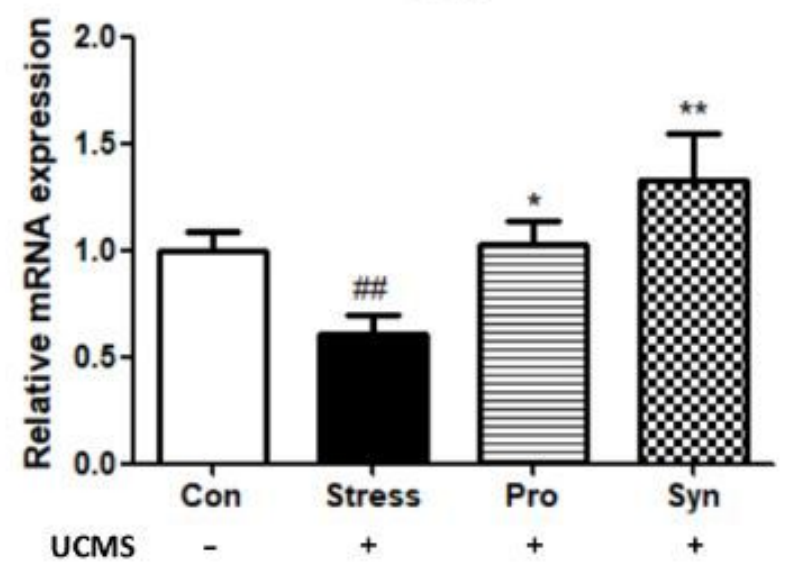

\section{Figure 2.}

\section{Figure 2}

Effects of probiotics and synbioticstreatments on the transcription of genes related to the HPGaxis in mouse brains during UCMS. Data represents the relative expression of $\mathrm{GnRH}, \mathrm{GnRHr}$, Fshb, and Lhbcompared to Gapdh. Data are expressed as the mean \pm SEM $(n=12)$ from three independent

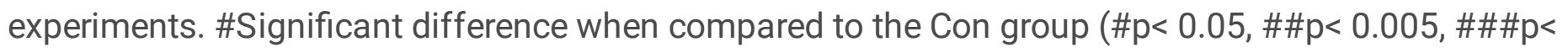
0.001). * Significant difference when compared to the Stress group ( $\left.p<0.05,{ }^{\star *} p<0.005,{ }^{\star * \star} p<0.001\right)$. 


\section{Adam5}

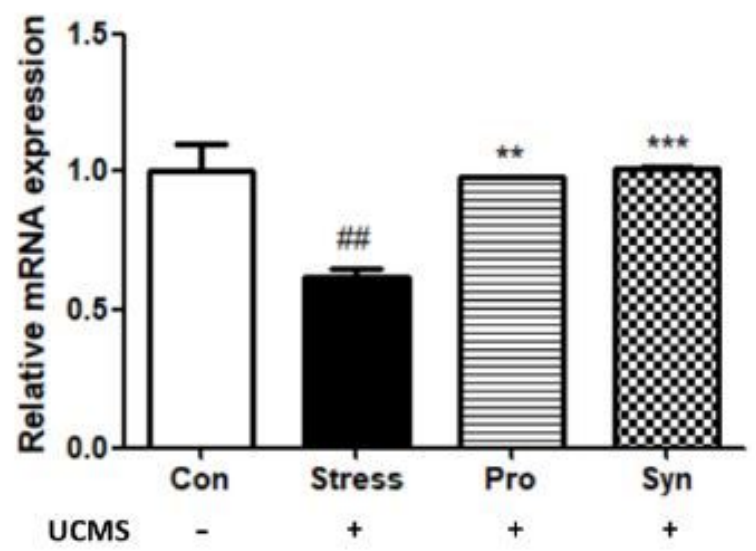

\section{Spam1}

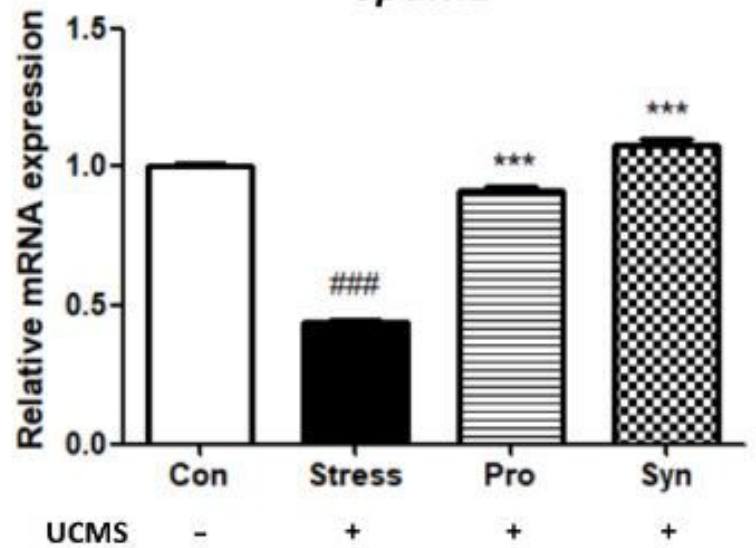

Adam29

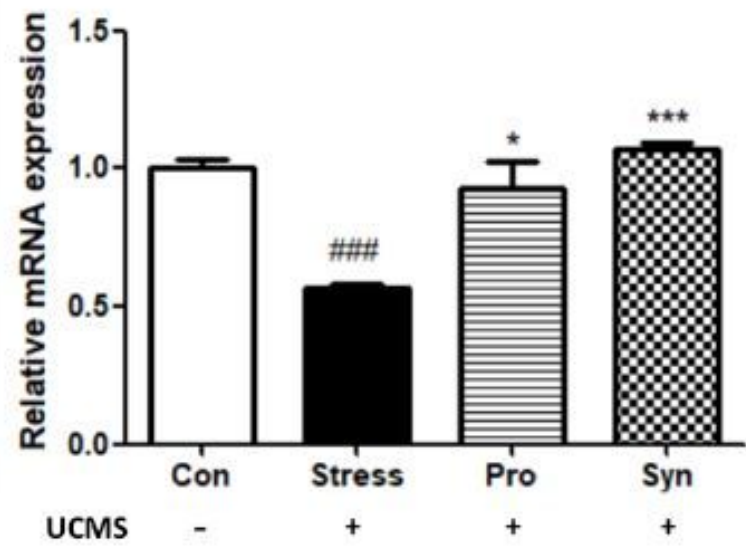

Tcfap2c

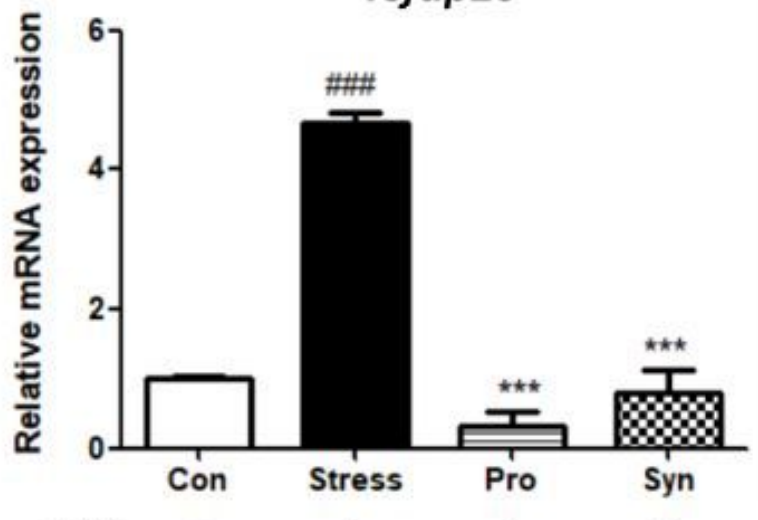

Figure 3.

Figure 3

Effects of probiotics and synbiotics treatments on the transcription of testicular development genes in mice testes under UCMS. Data represents the relative expression of Adam5, Adam29, Spam1, and Tcfap2ccompared to Gapdh. Data are expressed as the mean \pm SEM $(n=12)$ from three independent

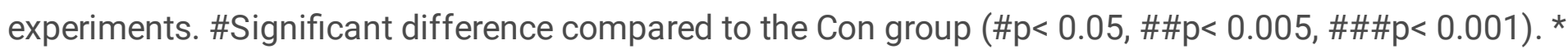
Significant difference compared to the Stress group $(* p<0.05$, $* * p<0.005, * \star \star p<0.001)$. 
Lhr

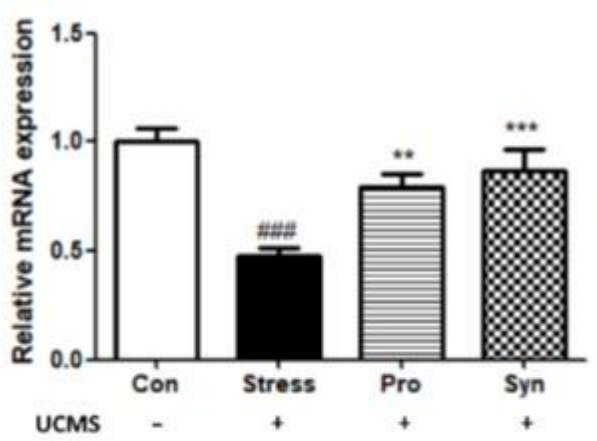

StAR

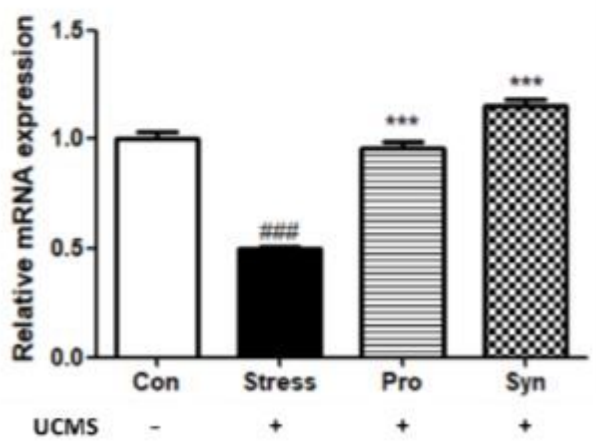

Egfr

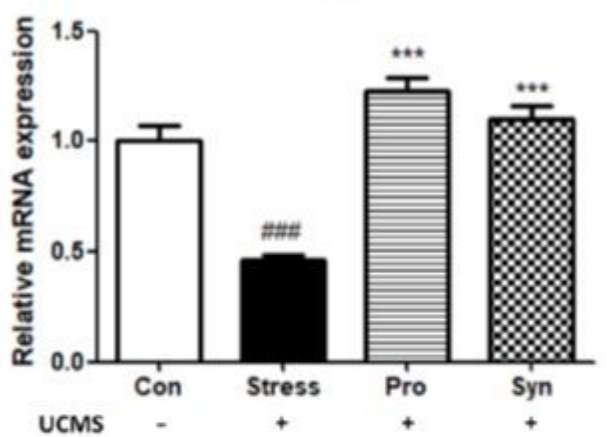

$\operatorname{Srd5a3}$

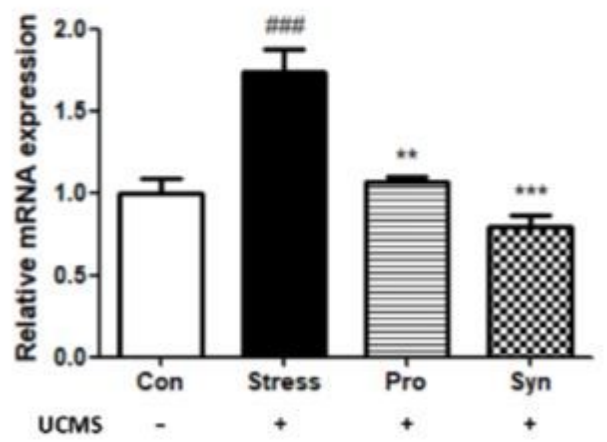

Inha

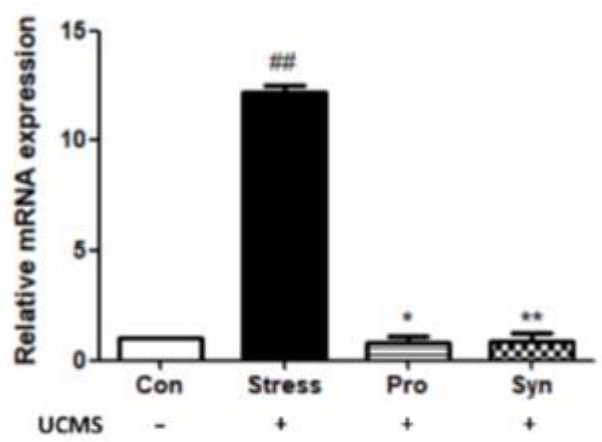

Cyp19a1

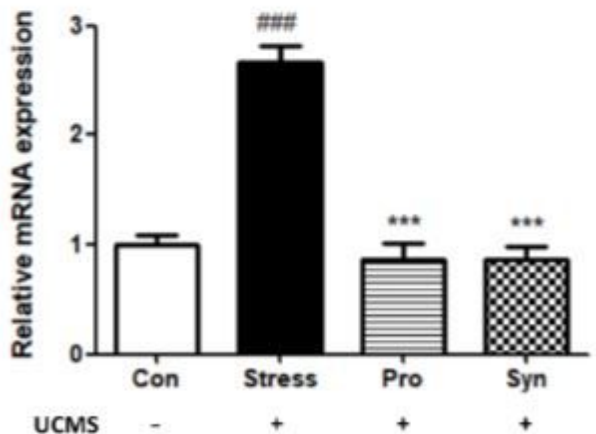

Figure 4.

Figure 4

Effects of probiotics and synbiotics treatments on the transcription of spermatogenesis and growth factor related genes in mice testes under UCMS. Data describes the relative expressions of Lhr, Egfr, Inha, StAR, Srd5a3, and Cyp19a1 compared to Gapdh. Data represent the mean \pm SEM $(n=12)$ from three independent experiments. \#Significant difference compared to the Con group (\#p $<0.05, \# \#<0.005$, $\# \# \# p<0.001)$. ${ }^{*}$ Significant difference compared to the Stress group $\left({ }^{*} p<0.05,{ }^{* \star} p<0.005,{ }^{* \star *} p<0.001\right)$. 


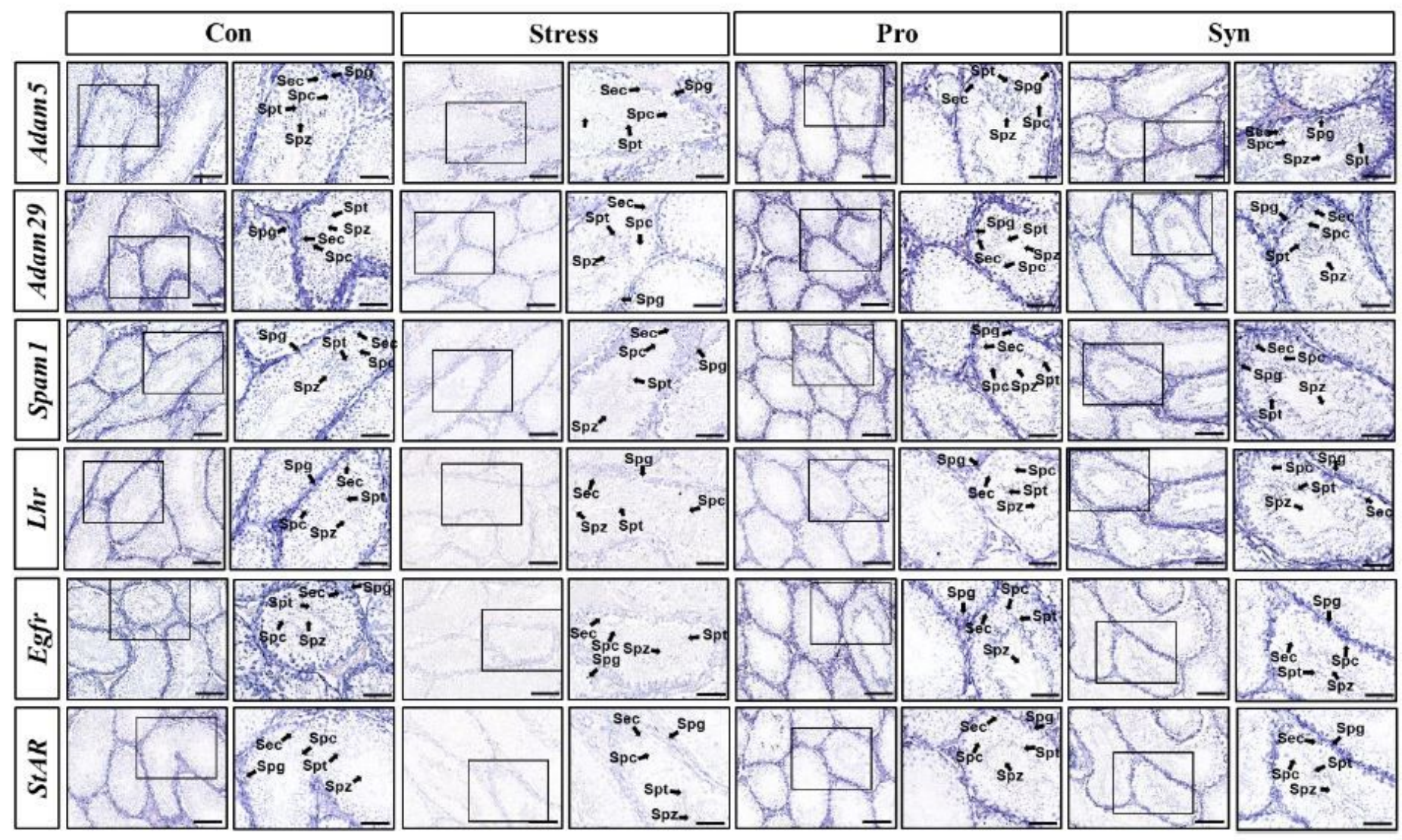

Figure 5.

\section{Figure 5}

In situ hybridization of Adam5, Adam29, Spam1, Lhr, Egfr, and StAR in mice testes. mRNA expression was analyzed using cross-sections from mice testes and antisense or sense target gene cRNA probes. $L$, Leydig cells; Spc, spermatocytes; Spg, spermatogonia; Spz, spermatozoa. Scale bar represents $10 \mu \mathrm{m}$. 